\title{
CLINIC AND THERAPEUTIC ASPECTS IN DUCTUS-DEPENDENT CONGENITAL HEART DEFECTS (PART II)
}

\author{
Alina-Costina Luca ${ }^{1}$, Andreea-Simona Holoc, Mirabela Subotnicu, \\ Constantin Iordache \\ ${ }^{I}$ Clinical Section of Pediatric Cardiology, "Sf. Maria”" Emergency Clinical Hospital for \\ Children, "Gr. T. Popa" University of Medicine and Pharmacy, Iasi
}

\begin{abstract}
The cardiac congenital malformations with duct dependent pulmonary circulation are cardiopathies with severe neonatal manifestations and quickly progressive hemodynamic degradation. The obvious semeiology at the birth requires an appropriate diagnostic and an early therapeutic management through the maintenance of the arterial channel permeability.

Keywords: duct dependent pulmonary circulation, right ventricular obstruction, arterial
\end{abstract} channel persistence, prostaglandin E1

The cardiac congenital malformations with duct dependent pulmonary circulation are affections characterized by the obstruction of the ejection tract of the right ventricle, at the level of which the left-right shunt is needed, in order to ensure the pulmonary circulation.

The cardiac malformations with duct dependent pulmonary circulation are:

- Pulmonary artery stenosis

- Pulmonary atresia with interrupted interventricular septum

- Fallot tetralogy - severe form

- Tricuspid valve atresia

- Ebstein's anomaly - severe form

- D-Transposition of the great arteries.

\section{PULMONARY ARTERY STENOSIS}

Within this malformation, one shall perform the severe obstruction of the right ventricle ejection tract. According to the localization, it can be valvular, subvalvular (infundibular) or supravalvular.

The critical pulmonary stenosis is associated to right ventricle hypertrophy or hypoplasia. The presence of a small communication at atrium level is needed so as to ensure a right-left shunt through the foramen ovale. The right-left shunt at atrium level and the arterial hypoxemia depend on the seriousness of the pulmonary stenosis and on the right ventricle hypoplasia (1).

When the stenosis is severe, the right-left shunt at atrium level can be massive, and the adequate flow at lung level is in accordance with the leftright shunt through the arterial channel. If the pulmonary stenosis is moderate, the blood flow at lung level is influenced to a small extent by the arterial channel closure (2).

In newborns with critical pulmonary stenosis, immediately after the birth appear tachypnea, cianosis and feeding difficulties. In case of the patients with severe pulmonary stenosis, we can notice the crowning of the precordial region; upon palpation, we can detect a ventricular impulse on the left margin of the stern and a systolic murmur in the left intercostals space II. The sound II is divided into two, the pulmonary component being delayed and diminished. In the severe forms, the sound II is weakly audible, the aortic component being cov- 
ered by the continuous murmur. At the level of the left intercostal space II-IV, we can identify an intense, rough, crescendo-decrescendo systolic murmur.

In the severe pulmonary stenosis, the EKG shows a right axial deviation and right ventricular hypertrophy. The two-dimension echocardiograph in the short axle section highlights the dome opening of the pulmonary valves, but also the measurement of the pulmonary valve ring. The color Doppler model helps at assessing the turbulent flow at the level of the pulmonary trunk.

\section{PULMONARY ATRESIA WITH INTACT INTERVENTRICULAR SEPTUM}

The ejection tract obstruction of the right ventricle is caused by the pulmonary valve atresia; thus, there is no communication between the pulmonary artery trunk and the right ventricle. The right-left shunt at atrium level depends on the leftright shunt at the arterial channel level. Therefore, the pulmonary arterial perfusion is produced through the arterial channel, the bronchial collaterals and permeable foramen ovale. Once with the gradual closure of the arterial channel, the pulmonary flow decreases even more and severe hypoxia and acidosis appear, which accentuate on a progressive basis $(3,4,5)$.

The anoxic crises, dyspnea with effort aggravated polypnea (nurse, cry) and right heart failure appear on a frequent basis.

The semeiology is obvious early in the 72 hours of life, the cianosis, initially moderate and then accentuated, becoming extreme as the arterial channel closes (4).

The clinical examination shows the systolic murmur, and upon the abdomen palpation, hepatomegaly. The cardiac auscultation shows that sound II is abolished. The following murmurs can appear: the aspiration murmur of the tricuspid valve failure and the systolodiastolic murmur of permeable arterial channel (3).

The electrocardiograph highlights the right electric axle $\left(+30^{\circ}\right.$ up to $\left.+90^{\circ}\right)$, right atrium hypertrophy and left ventricular hypertrophy (in case of marked hypoplasia of the right ventricle).

The two-dimensional echocardiograph is the election examination in the diagnostic of pulmonary atresia with intact interventricular septum. It highlights the atresia pulmonary valve, the tricuspid valve morphology, the right ventricle dimension and morphology. Moreover, color and continuous Doppler echocardiograph allow the tricuspid regurgitation, left ventricle pressure and arterial channel persistence assessment (16).

\section{FALLOT TETRALOGY-SEVERE FORM}

This form of disease belongs to the group of cardiac malformations with critical obstruction of the pulmonary circulation; the pulmonary blood flow is ensured by the opened arterial channel and/or by the aorto-pulmonary collaterals.

The clinical table is similar to the common form of Fallot tetralogy, but the cianosis appears in an early stage, there are frequent hypoxic bad conditions (the pulse oximetric values are equal in all limbs, with values of $70-80 \%$, in atmospheric oxygen). Sound II can be accentuated. The ejection systolic murmur is replaced by a left subclavicular continuous murmur determined by the presence of the permeable arterial channel $(6,7)$.

The electrocardiography highlights the electric axle deviated to the right, right ventricular or biventricular hypertrophy in case of heart failure.

The echocardiography highlights in the parasternal section of the long axle the visualization of the interventricular septum discontinuity, the determination of the extent to which the aorta is pressing-on the interventricular septum. If the aortic ring overlaps less than $50 \%$ of the left ventricle, we can establish the diagnostic of Fallot tetralogy, if it overlaps more than $50 \%$, the diagnostic is that of double outlet right ventricle. In the transversal sections, we can assess the discharge tract of the right ventricle and determine obstruction localization (subvalvular, valvular obstruction).

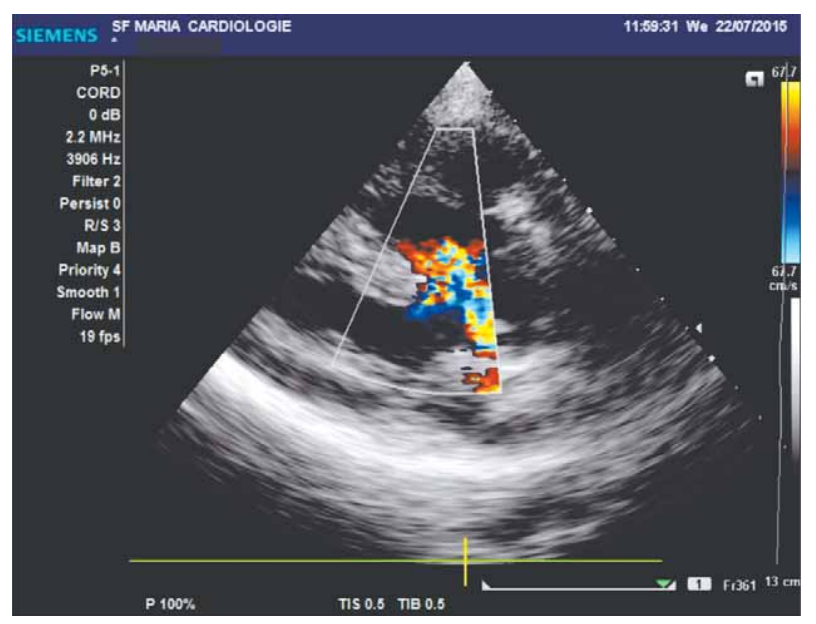

FIGURE 1. Patient C.S, 15 years old Echocardiograph - Fallot tetralogy

\section{TRICUSPID VALVE ATRESIA}

Represents a complex cyanogen congenital malformation characterized by the congenital absence 
(agenesis) of the tricuspid valve, so that the communication between the right atrium and ventricle is not possible.

The survival is possible only when there is a right-left shunt at atrium level which allows the vein blood from the vena cava to mix in the left atrium with the arterial blood came from the pulmonary veins; the pulmonary flow is reduced.

Moreover, the survival is possible when there is a left-right shunt which allows blood return in the pulmonary circulation through VSD or ACP.

After the vein blood is mixed with the arterial pulmonary one (came through the pulmonary veins) in the left atrium, it passes into the left ventricle. From the left ventricle, the blood gets partially in the aorta (and from here, by means of the permeable arterial channel, in the pulmonary artery), and partially, through the interventricular septum deficiency, in the right ventricle.

In the course of time, the pulmonary vessels resistance increases, appears the left ventricle volume overloading and the pulmonary high blood pressure, then the congestive heart failure (8).

From a clinical point of view, when the pulmonary flow is very low, there are hypoxic bad conditions and polyglobulia (1). When the pulmonary flow is high, appear constant dyspnea, recurrent respiratory infections and heart failure signs.

Upon the auscultation, we can notice a very strong sound II in the pulmonary focus (in the situation of the association to the arterial channel persistence); systolic murmur on the left margin of the stern (in association with the VSD) or left subclavicular continuous murmur (in the arterial channel persistence) (9).

The cardio-pulmonary radiograph highlights an increased cardiothoracic index in case of right or left ventricle dilatation, the pulmonary circulation appearance varying according to the pulmonary flow. The electrocardiogram shows right atrium hy- pertrophy (wave amplitude $\mathrm{P}>2.5 \mathrm{~mm}$ in DII is present in $75 \%$ of the patients), left ventricular hypertrophy with QRS axle deviation to the left.

The transthoracic echocardiograph establishes the diagnostic, identifying the absence of the right atrioventricular connection, atresia type, the localization and dimension of the interatrial and interventricular communication.

\section{EBSTEIN'S DISEASE - SEVERE FORM}

Congenital heart malformation characterized by diverse anomalies of the tricuspid valve (low implantation of the septum and posterior laminas), with right atrium volume increase and right ventricle volume decrease (most of it being atrialized).

The right-left shunt at atrium level is the one reflecting the hypoxemia degree. The increased vascular resistances lead to the right ventricle postcharge increase, as the right-left shunt becomes massive and implicitly the hypoxemia becomes severe (there are frequent hypoxic crises), with different degrees of cianosis, dyspnea and tachycardia (10).

When the communication through the arterial channel closes, the hypoxemia is accentuated, determining respiratory acidosis and death. In the absence of interatrial communications appears the right heart failure with hepatomegaly.

From a clinical point of view, in the severe forms, cianosis appears early in the first days of life; sound II is split into two, 3 or 4-step rhythm; pansystolic tricuspid regurgitation murmur, systolic murmur, stasis hepatomegaly (11).

On the electrocardiogram there are signs of right atrium supra solicitation: high acute $\mathrm{P}$ waves, supraventricular rhythm troubles, delta wave presence indicates the existence of the WPW syndrome (Fig. 2) (12).

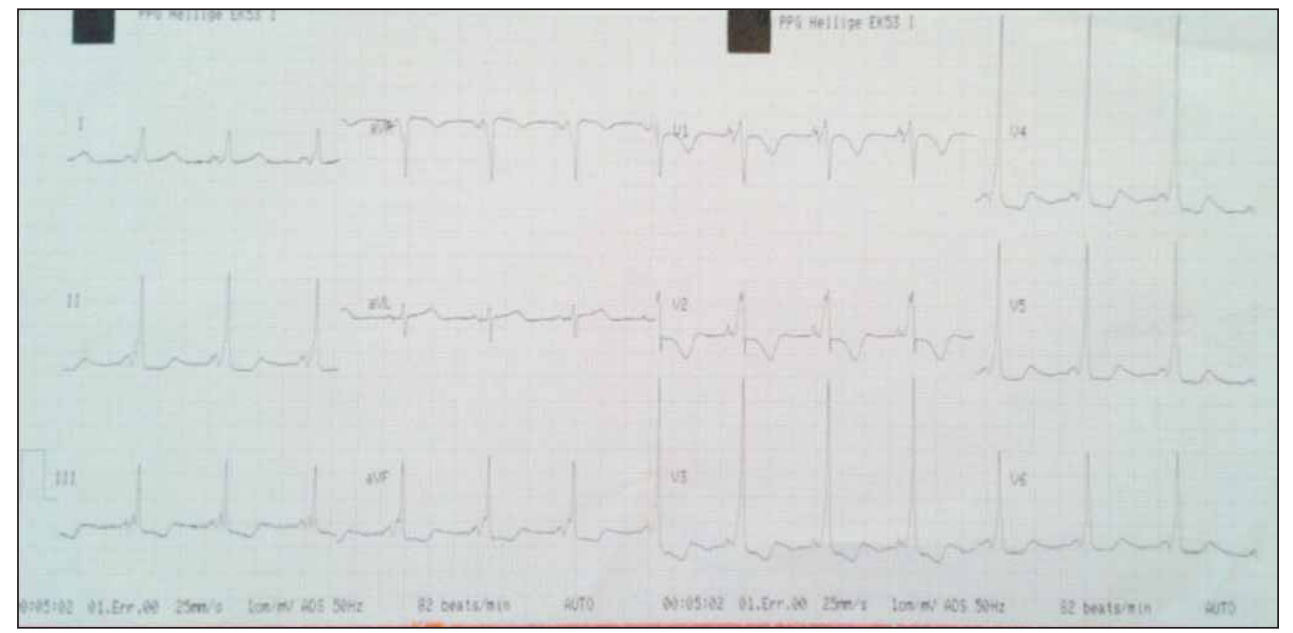

FIGURE 2. Patient S.A.G, 11 days: the electrocardiogram in Ebstein's disease: delta wave present in DI, DII, V3, V4, V5 (WPW syndrome). 
The transthoracic echocardiograph - apical 4 rooms is the election section in Ebstein's anomaly diagnostic. We assess the atrialization degree of the right ventricle, the displacement and distortion of the tricuspid laminas, but also the closeness degree of the anterior laminas of the tricuspid valve at the right ventricle. By means of color Doppler one can determine the presence and size of the interatrial shunt (Fig. 3).
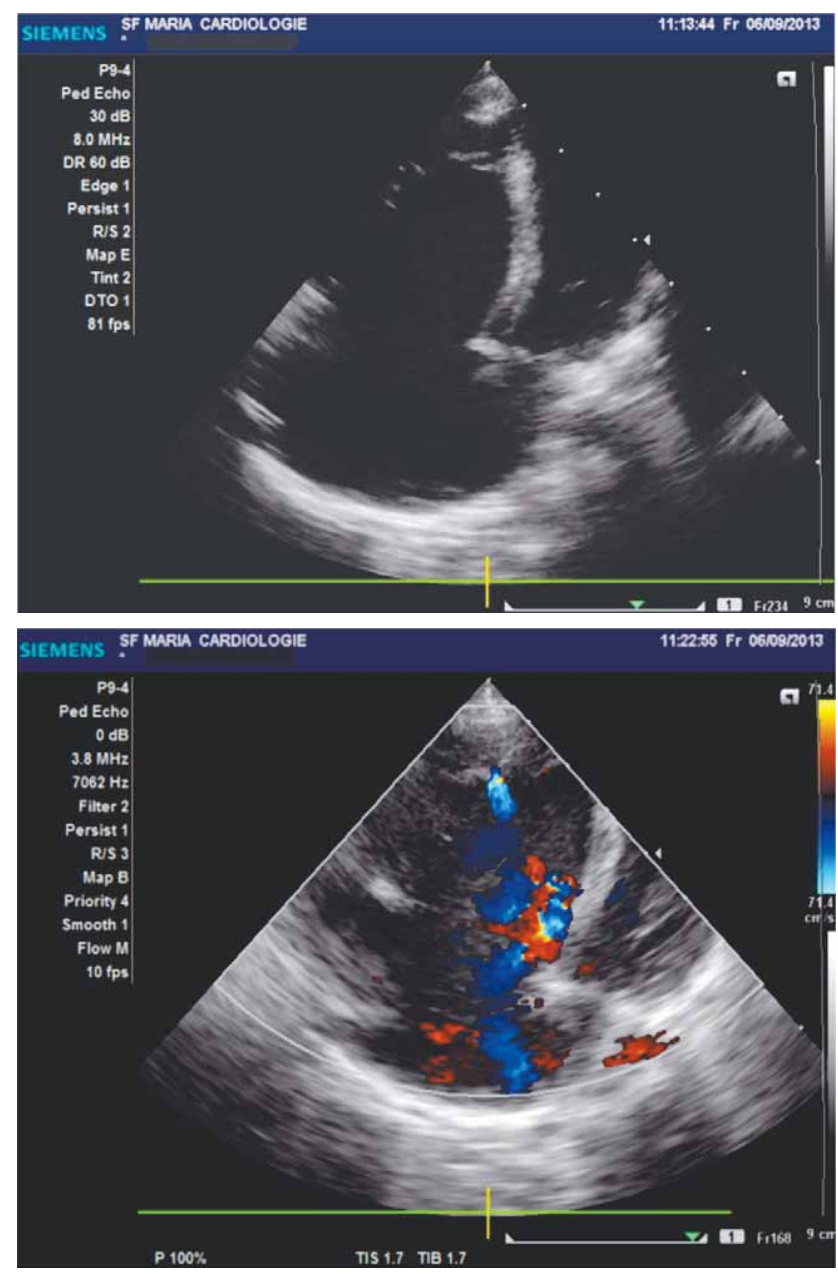

FIGURE 3. Patient S.A.G, 11 days: echocardiography apical 4 rooms - Ebstein's anomaly - severe form

\section{D-TRANSPOSITION OF THE GREAT ARTERIES}

Is defined by the ventricle-atrium discordance, within the meaning that the aorta emerges from the morphological right ventricle and the pulmonary artery emerges from the morphological left ventricle.

Two elements are particular:

- The aorta is located in anterior position as compared to the pulmonary artery

- There is an atrioventricular concordance.

\section{Hemodynamics}

The two circulations, systemic and pulmonary are not serial, but parallel, namely the systemic venous blood reaches in the right atrium, then in the right ventricle and then in the aorta, and the oxygenated blood from the lungs reaches in the left atrium, then in the left ventricle, then he passes in the pulmonary artery.

The child can survive if there are shunts between the two circulations through the arterial channel and the foramen ovale. After the birth, the pulmonary resistances decrease and, through the arterial channel, the arterial desaturated blood passes from the aorta in the pulmonary artery. Therefore, appears a severe hypoxia with oxygen saturations of $30-60 \%$ and a pulmonary pressure of $15-40 \mathrm{~mm}$ $\mathrm{Hg}$. Only through the existence of a permeable foramen ovale, the oxygenated blood from the pulmonary veins reaches in the left atrium and then in the right atrium and then in the systemic circulation (bidirectional shunt), preventing therefore cianosis occurrence, but leading to the early installation of the congestive heart failure $(13,14)$.

Other associated defects needed for the survival are the interatrial and interventricular septum defects.

\section{Clinically}

Initially, there are signs of early pulmonary atrium high blood pressure and cianosis (rebel to oxygen administering), whose intensity depends on the communications between the two parts of the heart.

When ACP is large and VSD is associated too, the clinical picture is that of congestive heart failure (stable dyspnea, tachypnea, transpirations, grunt, rough cry).

According to the associated heart malformation, the systolic murmur can be intense (interventricular septum defficiency) or systolo-diastolic (arterial channel persistence). The ventricular dilatation is associated to the protodiastolic gallop (sound III), and the atrium dilatation with presystolic gallop (15).

\section{Paraclinically}

The electrocardiogram shows axle deviation to the right, right ventricular hypertrophy (ample $\mathrm{R}$ in $\mathrm{V} 1$ and deep S in V6). The cardio-pulmonary radiograph shows a narrowed mediastinum, cardiac silhouette with classical ovoid shape of "egg on a string" and accentuated pulmonary pattern.

The transthoracic echocardiograph in the parasternal section of the short axle at the level of the 
great arteries highlights the great arteries with parallel disposal, the aorta located in anterior position and to the right as compared to the pulmonary artery. The Doppler ultrasound assesses the pulmonary transvalvular gradient.

\section{THE PHARMACOLOGICAL TREATMENT OF THE CARDIAC CONGENITAL MALFORMATIONS WITH DUCT DEPENDENT PULMONARY CIRCULATION}

\section{Recommendations}

Careful oxygen administering, so as to avoid the arterial channel closure, maintaining the saturations in the right hand of more than $75 \%$, in order to decrease the pulmonary vascular resistance.

The maintenance of arterial channel permeability through the administering of Prostaglandin E1: 0.15-0.20 $\mu \mathrm{g} / \mathrm{kgc} / \mathrm{min}$ with the supplementation $0.20 \mu \mathrm{g} / \mathrm{kgc} / \mathrm{min}$ every 15 minutes until the effect is obtained (the half-time is of 30 seconds, so the continuous administering of the perfusion is indicated); the careful monitoring of the neuro-psychic status, of the heart rate, of the blood pressure, of the respiratory status, the EKG tract during the perfusion is very important, because of side effects occurrence: convulsions, bradipnea, low blood pressure, tachycardia, II-nd degree atrioventricular block, supraventricular tachycardia, ventricular fibrillation, cardio respiratory arrest (17).

When the pulmonary circulation is overstrained (the pressure is normal or high), we recommend angiotensin converting enzyme inhibitors - Captopril $1 \mathbf{~ m g} / \mathbf{k g c}(18,19)$.

If the blood pressure is low, we recommend phosphodiesterase inhibitors - Sildenafil 0.5-2 mg/ kge/dose.
Diuretics are associated - Spironolactone 1 $\mathrm{mg} / \mathrm{kgc}$ in 1-3 uptakes; in the severe forms of congestive heart failure Furosemide $1 \mathrm{mg} / \mathbf{k g c} / \mathbf{d o s e}$ is associated, up to a diuresis higher than $3 \mathrm{ml} / \mathrm{kcg} /$ hour, dose which shall be repeated every 6-12 hours; in the absence of the effect, the dose shall be repeated in 2 hours.

Shall be administered up to the normal volumeexpander (physiological serum $0.9 \%$ or lactate Ringer) - 10 ml/kgc intravenous.

The severe metabolic acidosis shall be treated with sodium hydrogen carbonate $4.2 \%$ (in a quantity of $\mathbf{2} \mathbf{~ m E q} / \mathbf{k g c} / \mathbf{d o z a ̆}$ ) intravenous, very slowly, at adequate ventilation.

The administering of prostaglandin E1 for the maintenance of the opening of the arterial channel. The initial dose is of $\mathbf{0 . 0 5 - 0 . 1 ~} \mathbf{~ m e g} / \mathbf{l g c} / \mathbf{m i n}$.

\section{CONCLUSIONS}

The critical semeiology present at birth and the progressive deterioration impose an early diagnostic in order to prevent complications. The echocardiograph is an important paraclinical examination in the diagnostic of the cardiac congenital malformations with dependent pulmonary circulation, being compulsory for the child hospitalization and the cardiac deterioration monitoring. Therefore, a careful clinical examination, diagnostic confirmation through paraclinical methods as accurate as possible, the maintenance of the arterial channel permeability and the referral to a specialized center for the surgical intervention are essential in managing these cases.

\section{REFERENCES}

1. Mouledoux J.H, Walsh W.F. Evaluating the Diagnostic Gap: Statewide Incidence of Undiagnosed Critical Congenital Heart Disease Before Newborn Screening With Pulse Oximetry. Pediatric Cardiology 2013; 34:1680-1686.

2. Costello J.H., Laussen P.C. Cyanotic CHD Lesions with Decreased Pulmonary Blood Flow. "In: Wheeler D.S, Wong H.R, Shanley T.P. Pediatric Critical Care Medicine: Volume 2: Respiratory, Cardiovascular and Central Nervous Systems". Second Edition. Springer. $2014: 366-373$.

3. Chubb H., Daubeney P.E. Pulmonary Atresia with intact ventricular septum. "In: Moller J.H., Hoffman J.I. Pediatric Cardiovascular medicine", Second edition, Wiley - Blackwell. 2012: 572-584.

4. Roman K.S., Fouron J.C., ONii M., et.al. Determinants of Outcome in Fetal Pulmonary Valve Stenosis or Atresia With Intact Ventricular

Septum. The American Journal of Cardiology, Volume 99, Issue 5, 2007:0699-703.

5. Guleserian K.J., Armsby L.B., Thiagarajan R.R., del Nido P.J. Natural history of pulmonary atresia with intact ventricular septum and right-ventricle-dependent coronary circulation managed by the single-ventricle approach. Ann Thorac Surg. 2006; 81(6):2250-2257.

6. Alson D. Inaba. Congenital Disease. "In: Jill M. Bare. Pediatric Emergency Medicine". Saunders; 1 edition, 2007: 283-286.

7. Sin Weon Yun. Congenital heart disease in the newborn requiring early intervention. Korean J Pediatr 2011; 54(5): 183-191

8. Michael L. Epstein. Tricuspid Atresia, Stenosis, Regurgitation, and Uhl'Anomaly. "In: De Hugh D. Allen, David J. Driscoll, Robert E. Shaddy, Timothy F. Feltes Moss \& Adams' Heart Disease in Infants, Children, and Adolescents: Including the Fetus and Youg Adult", Eight 
edition, Lippincott Wiliams and Wilkins, a Wolkers KluWER, 2013: 877-888

9. R.M. Freedom, L.N. Benson. Tricuspid Atresia. "In: Robert M. Freedom, Leland N. Benson, Jeffrey F. Smallhorn. Neonatal Heart Disease, Springer - Verlang, 2012 : 229-246

10. Kipps A.K., Graham D.A., Lewis E., Marx G.R., Banka P., Rhodes J. Natural history of exercise function in patients with Ebstein anomaly: A serial study. Am Heart J. 2012 Mar. 163(3):486-91

11. J.M. Costello, P.C. Laussen. Congenital heart disease: Cyanotic lesions with decreased pulmonary blood flow. "In: Derek S. Wheeler, Hector R. Wong, Thomas P. Shanley Pediatric Critical Care Medicine: Basic Science And Clinical Evidence". Springer. 2007: 697-690

12. Oxenius A., Attenhofer Jost C.H., Prêtre R. Management and outcome of Ebstein's anomaly in children. Cardiol Young. 2013; 23(1):27-34

13. Squarcia U., Macchi C. Transposition of the great arteries. Curr Opin Pediatr. 2011; 23(5):518-22

14. Unolt M., Putotto C., Silvestri L.M. et.al. Transposition of great arteries: new insights into the pathogenesis. Front Pediatr. 2013; 1:11.

15. Rao P.S. Diagnosis and management of cyanotic congenital heart disease: part I. Indian J Pediatr. 2009 Jan. 76(1):57-70.
16. Burch T.M., Mizuguchi K.A., Wesley M.C., Swanson T.M., Dinardo J.A. Echocardiographic features of pulmonary atresia with intact ventricular septum. Anesth Analg. 2008 Nov. 107(5):1509-11.

17. Huang F.K., Lin C.C., Huang T.C., Weng K.P., Liu P.Y., Chen Y.Y., Wang H.P., Ger L.P., Hsieh. Reappraisal of the prostaglandin E1 dose for early newborns with patent ductus arteriosus-dependent pulmonary circulation. Pediatr Neonatol. 2013; 54(2):102-6.

18. I.B. Vijayalakshmi, P. Syamasundar Rao, Reema Chugh. A Comprehensive Approach to Congenital Heart Diseases. JP Medical Ltd 2013, 5:89-93

19. Helmut Baumgartner, Philipp Bonhoeffer, Natasja M.S. De Groot, Fokko de Haan, John Erik Deanfi eld et al. ESC Guidelines for the management of grown-up congenital heart disease (new version 2010). The Task Force on the Management of Grown-up Congenital Heart Disease of the European Society of Cardiology (ESC). European Heart Journal, 2010. 